\title{
Prostatectomía radical extraperitoneal laparoscópica: evolución en el tiempo y resultados actualizados
}

\author{
Stolzenburg JU*, Rabenalt R*, Do M *, Jiménez Cruz F**, Liatsikos EN*** \\ *Servicio de Urología. Universidad de Leipzig. Alemania. ${ }^{* *}$ Servicio de Urología. Hospital Universitario La Fe. \\ Valencia. ***Servicio de Urología. Hospital Universitario de Patras. Grecia.
}

Actas Urol Esp. 2006; 30 (6): 556-566

\section{RESUMEN}

PROSTATECTOMÍA RADICAL EXTRAPERITONEAL LAPAROSCÓPICA: EVOLUCIÓN EN EL TIEMPO Y RESULTADOS ACTUALIZADOS

La prostatectomía radical laparoscópica ha sido establecida y adoptada por centros urológicos especializados de todo el mundo como el método fundamental de tratamiento quirúrgico del cáncer de próstata localizado. Los urólogos mantienen una búsqueda constante de desarrollo de modificaciones y refinamientos técnicos, para reducir la morbilidad y mejorar el resultado clínico y de calidad de vida.

En 2002, comunicamos inicialmente nuestra técnica y experiencia con la prostatectomía radical endoscópica totalmente extraperitoneal (PRETE). En este artículo de revisión presentamos la evolución de la técnica y los últimos resultados.

Palabras clave: Prostatectomía radical extraperitoneal. Técnica. Laparoscopia. Cáncer de próstata.

\section{ABSTRACT \\ LAPAROSCOPIC EXTRAPERITONEAL RADICAL PROSTATECTOMY: EVOLUTION IN TIME AND UPDATED RESULTS}

Laparoscopic radical prostatectomy has been established and adopted by specialized urologic centers around the world as the primary approach to the surgical management of localized prostate cancer. Urologists are on a continuous search for development of technical modifications and refinements, to reduce morbidity and ameliorate clinical and quality of life outcome.

In 2002 we have initially reported our technique and experience with the totally extraperitoneal endoscopic radical prostatectomy (EERPE). In the present review paper we present the evolution of the technique and the latest results.

Key words: Extraperitoneal radical prostatectomy. Technique. Laparoscopy. Prostate cancer.

$\mathrm{L}$ a prostatectomía radical laparoscópica (PRL) ha sido establecida y adoptada por centros urológicos especializados de todo el mundo como el método fundamental de tratamiento quirúrgico del cáncer de próstata localizado. Datos recientes sugieren que no hay diferencias significativas entre la prostatectomía retropúbica radical abierta estándar y la PRL en cuanto a resultados funcionales y control del cáncer a corto plazo (márgenes quirúrgicos positivos y progresión del PSA).
Además, la PRL aporta una visión ampliada excelente de la anatomía pélvica, un tiempo de cateterización más corto y baja tasa de complicaciones intra y posoperatorias ${ }^{1-6}$.

Raboy et al. describieron el primer caso de prostatectomía radical laparoscópica extraperitoneal ${ }^{7}$ y Bollens et al. describieron la primera serie de casos usando esta técnica ${ }^{8}$. En 2002, comunicamos nuestra técnica y experiencia inicial con la prostatectomía radical laparoscópica totalmente extra- 
peritoneal $(\mathrm{PRETE})^{9}$. En 2003, publicamos los resultados obtenidos en los 70 primeros $\operatorname{casos}^{10}$. Desde entonces, hemos mejorado la técnica de forma continua, incluyendo la conservación de nervios para mantener la potencia sexual (PRETEcn) ${ }^{11}$. En este trabajo, presentamos la evolución de la técnica y los últimos resultados oncológicos y funcionales después de 700 procedimientos.

\section{LA TÉCNICA}

Ya se ha descrito previamente la técnica inicial de PRETE ${ }^{9-11}$. Al aumentar nuestra experiencia en la realización de PRETE, pudimos desarrollar un planteamiento de conservación de nervios (PRETEcn) que aplicamos de forma habitual en pacientes seleccionados. Además, se ha desarrollado una técnica que conserva el ligamento puboprostático, comprobándose la recuperación más precoz de la continencia después de la cirugía, sin comprometer el resultado oncológico final del procedimiento.

Estas modificaciones y la técnica de conservación de nervios se describen en detalle a continuación. Se resumen brevemente los pasos principales de la PRETE, como se comunicaron con anterioridad.

\section{Posicionamiento del paciente}

La PRETE suele realizarse con el paciente bajo anestesia general. Se coloca a los pacientes en decúbito supino con las piernas ligeramente separadas (Fig. 1). La torre de laparoscopia se coloca al pie de la mesa de operaciones y al paciente en decúbito supino con una inclinación de la cabeza de $20^{\circ}$ hacia abajo. Al acrecentarse nuestra experiencia, nos dimos cuenta de que no había necesidad de una posición Trendelenburg extrema y que una inclinación hacia debajo de la cabeza de $10^{\circ}$ era suficiente para realizar el procedimiento, porque el intestino no interfería con este procedimiento. Además, incluso tiempos quirúrgicos largos en casos difíciles pueden controlarse sin limitaciones cardiopulmonares.

\section{Colocación del trócar}

El primer paso en el procedimiento es crear un espacio preperitoneal y la colocación del primer trócar. Se realiza una incisión de $15 \mathrm{~mm}$ en el pliegue infraumbilical inmediatamente lateral a

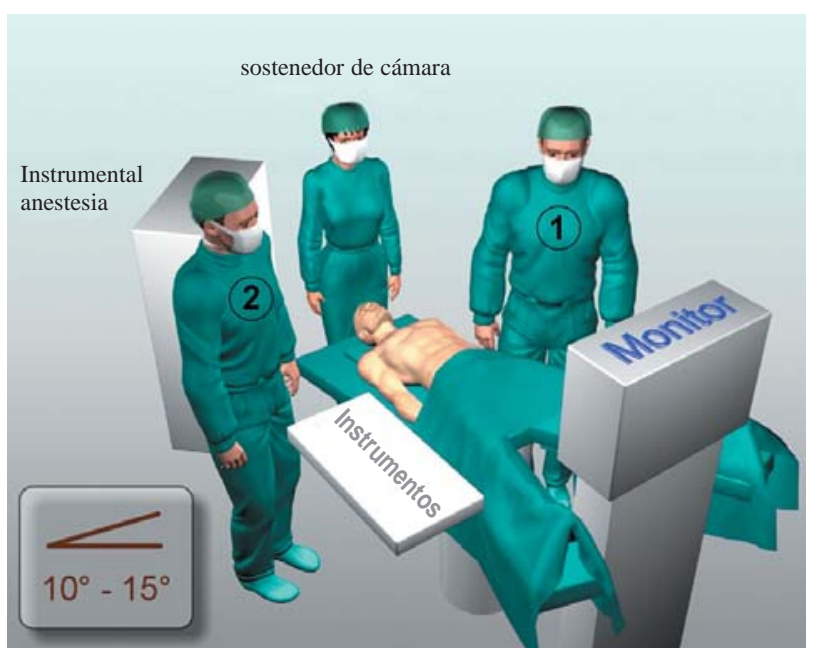

FIGURA 1. Configuración del quirófano para PRETE. El cirujano se sitúa a la izquierda (N. 1) y el ayudante a la derecha (N. 2) del paciente. El soporte de la cámara se coloca detrás de la cabeza del paciente. Todos tienen una visión directa sobre el monitor que se coloca entre las piernas abiertas del paciente.

la línea media y se lleva hacia abajo a la fascia del recto anterior. Se corta horizontalmente la fascia del recto anterior, exponiendo el músculo recto. Se separan verticalmente las fibras del músculo recto mediante disección roma, exponiendo la fascia del recto posterior. El espacio entre el músculo recto y el vaina posterior del recto se amplía de forma roma mediante disección con el dedo en la dirección del espacio preperitoneal (Fig. 2). La vaina posterior del recto está ausente por debajo de la línea arqueada. Se introduce un trócar de balón por encima de la vaina del recto posterior y se insufla bajo control visual directo (Fig. 3). Los vasos epigástricos y el arco púbico son las principales referencias durante la dilatación con balón. Se retira el trócar de balón y se colocan suturas de sujeción a ambos lados de la incisión de la fascia del recto. Se introduce y se fija un trócar óptico (de tipo Hassan). Después de la insuflación con $\mathrm{CO}_{2}$ (12 mmHg), se coloca el segundo trócar de $5 \mathrm{~mm}$ dos dedos a la izquierda, lateral a la línea media (en una proporción 1/3 a 2/3 del ombligo al arco púbico) utilizando este puerto se diseca bajo visión el espacio preperitoneal para rechazar el peritoneo. Se coloca un trócar de 5 $\mathrm{mm}$ medial a la espina ilíaca superior anterior derecha. Se coloca otro trócar de $5 \mathrm{~mm}$ en la línea pararrectal derecha, teniendo cuidado de evitar lesionar los vasos epigástricos. Se coloca el trócar 


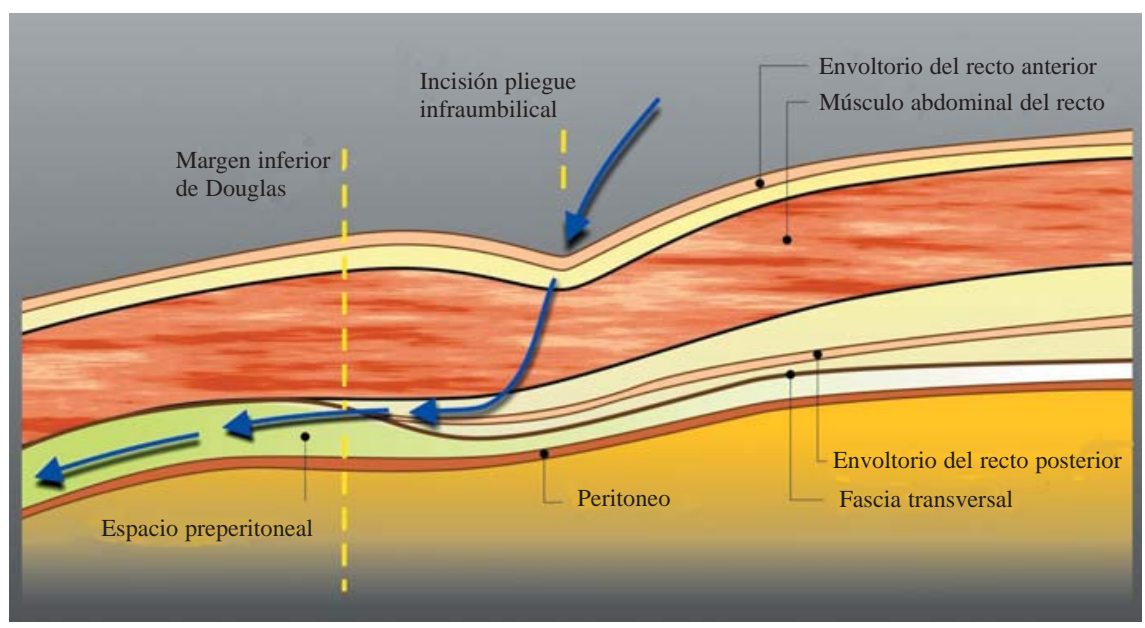

FIGURA 2. Disección del espacio preperitoneal.

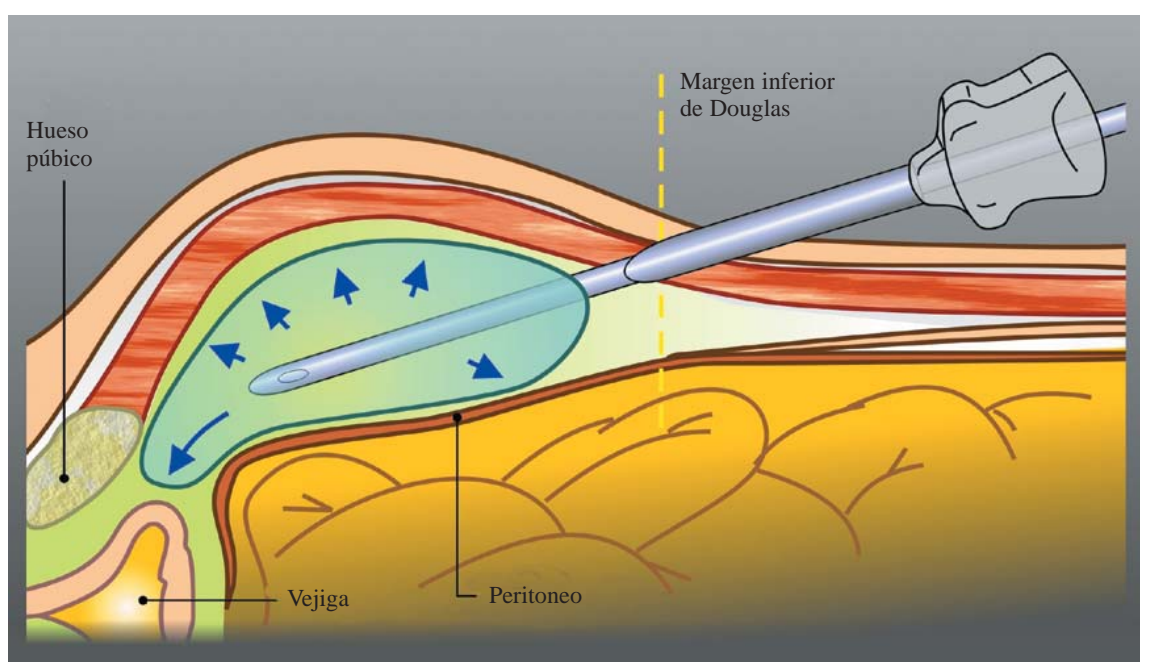

FIGURA 3. Inserción del trócar de balón e insuflación que crea el espacio de trabajo retroperitoneal.

final de $12 \mathrm{~mm}$ aproximadamente a tres dedos medial a la espina ilíaca superior anterior izquierda (Fig. 4). En pacientes muy obesos o muy altos, todos los trócares deben colocarse 1-3 cm caudalmente para un acceso óptimo al espacio retropúbico.

Disección de ganglios linfáticos pélvicos

Cuando está indicado, se realiza disección de los ganglios linfáticos pélvicos como procedimiento de estadificación, incluyendo los ganglios linfáticos del área entre la bifurcación de la arteria ilíaca común (borde craneal), los vasos ilíacos y el cordón espermático (borde lateral), el hueso púbico (borde caudal) y el nervio obturador (borde posterior).
Exposición de la próstata y el cuello vesical e incisión de la fascia endopélvica

El primer paso del procedimiento es la disección del espacio de Retzius. Se exponen la superficie anterior del cuello de la vejiga, la superficie anterior de la próstata y la fascia endopélvica y se separa suavemente el tejido graso suprayacente a estas estructuras. A menudo, hay una rama superficial del complejo de la vena dorsal profunda que discurre a lo largo de la cara anterior de la próstata y se divide en el cuello vesical en dos ramas. Se fulgura esta vena con pinzas bipolares y se corta. Luego, se incide la fascia endopélvica a ambos lados, exponiendo las fibras del músculo elevador del ano.

Corte de los ligamentos puboprostáticos y ligadura del plexo de la vena dorsal

Se cortan ambos ligamentos (Fig. 5). Después de este paso, la uretra y el complejo de la vena dorsal pueden visualizarse fácilmente a nivel del vértice prostá-

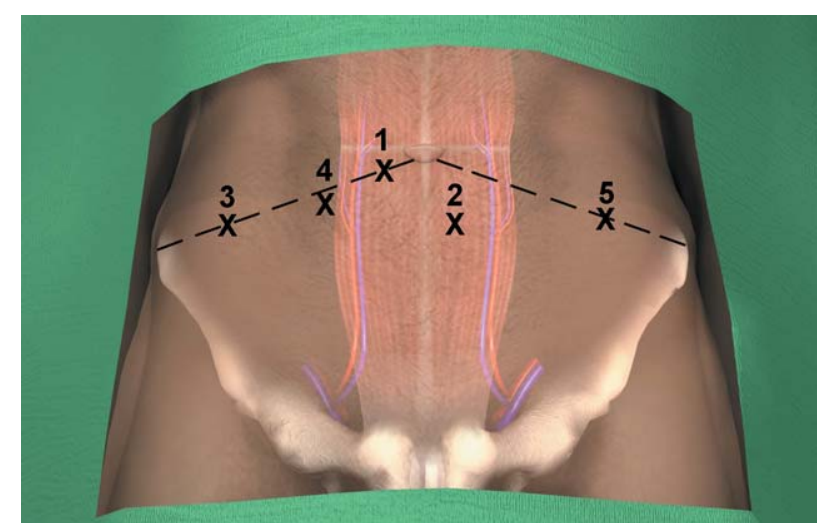

FIGURA 4. Colocación del catéter para PRETE (secuencia de colocación de trocares para PRETE). Observe el trayecto de los vasos epigástricos a ambos lados. El trócar $n^{\circ} 2$ debe insertarse medialmente y el trócar $n^{\circ} 3$ debe insertarse lateralmente a los vasos epigástricos. El trócar no 3 a menudo está muy próximo a los vasos. 
tico. Luego el asistente retrae la próstata proximalmente para conseguir un buen acceso al plexo de Santorini y una adecuada maniobrabilidad de la aguja. El plexo de Santorini es ligado con Vicryl 0 en una aguja CT-1 (ligeramente rectificada hacia arriba) mediante el paso selectivo de la aguja por debajo del plexo de izquierda a derecha (Fig. 6).

\section{Disección del cuello vesical}

El cuello vesical puede identificarse después de la retirada de todo el tejido graso perivesical adherido, que se solapa sobre la próstata en forma de triángulo. La disección comienza en la

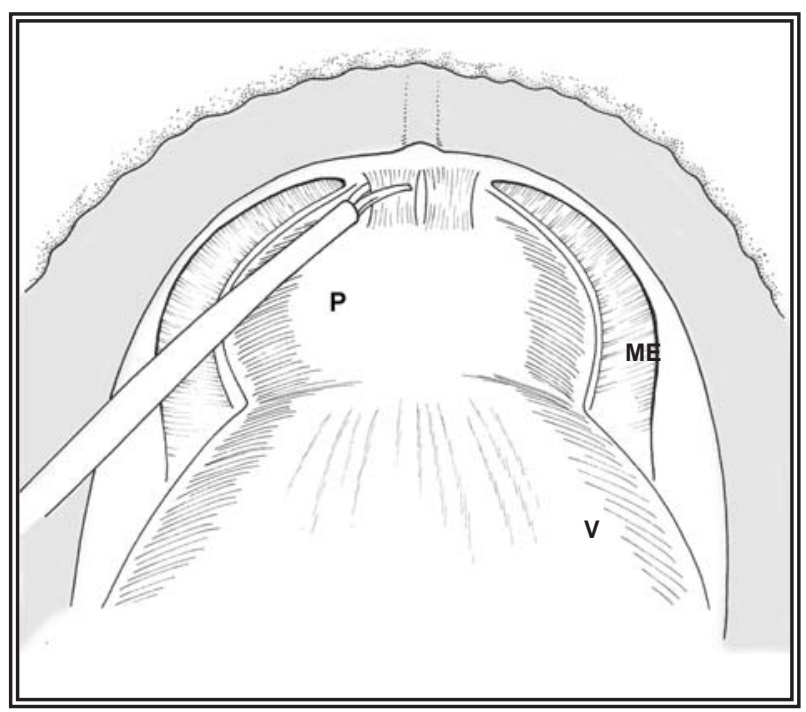

FIGURA 5. Disección de los ligamentos puboprostáticos. Se muestra la fascia endopélvica abierta y se ven las fibras del músculo elevador del ano.

$P=$ próstata, $M E=$ músculo elevador del ano, $\mathrm{V}=$ vejiga .

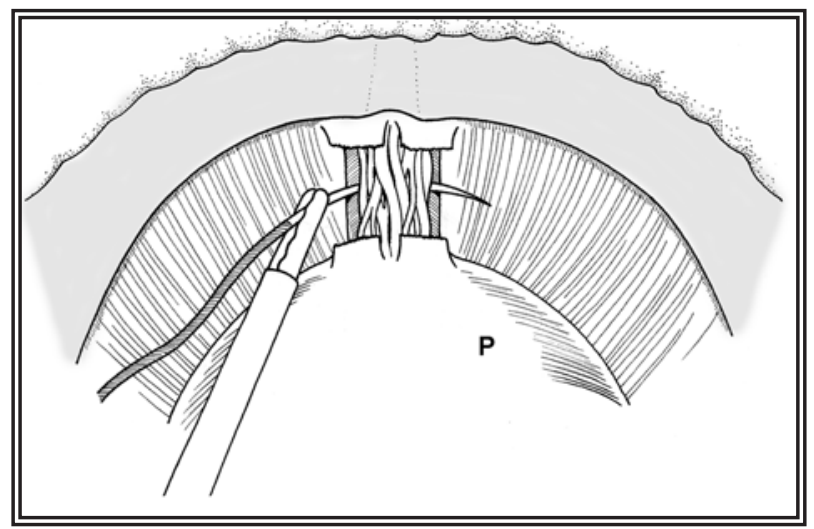

FIGURA 6. Ligadura del plexo de Santorini (Vicryl-o en una aguja CT-1) de izquierda a derecha.

$P=$ próstata. posición de las 12, en la punta de este triángulo (Fig. 7). La palpación con el fórceps y la movilidad del balón del catéter, pueden ayudar a identificar el límite entre el cuello móvil de la vejiga y la próstata sólida, en casos difíciles. Se amplía la disección del cuello de la vejiga desde la posición de las 10 a la de las 2 y se diseca la musculatura longitudinal del cuello de la vejiga. Se incide en el cuello de la vejiga y el asistente tira hacia arriba el catéter-balón deshinchado hacia el espacio retropúbico bajo tensión continua. Ahora se continúa la disección en la dirección lateral, en un plano entre el cuello de la vejiga y la próstata.

En procedimientos de conservación de nervios, es importante cortar la fascia delgada suprayacente a la cara anterior de la próstata, para movilizar ambos haces neurovasculares antes de la preparación y disección de la parte dorsal del cuello de la vejiga. Luego, se diseca completamente el cuello vesical. Se tiene cuidado de llevar la disección en el plano correcto entre la próstata y el cuello vesical para evitar cualquier perforación intraprostática. Primero se divide completamente el cuello vesical entre las posiciones de las 5 y las 7 en punto. Se visualizan las porciones ampulares de los conductos deferentes como referencias anatómicas. La disección se extiende bilateralmente de forma roma y cortante.

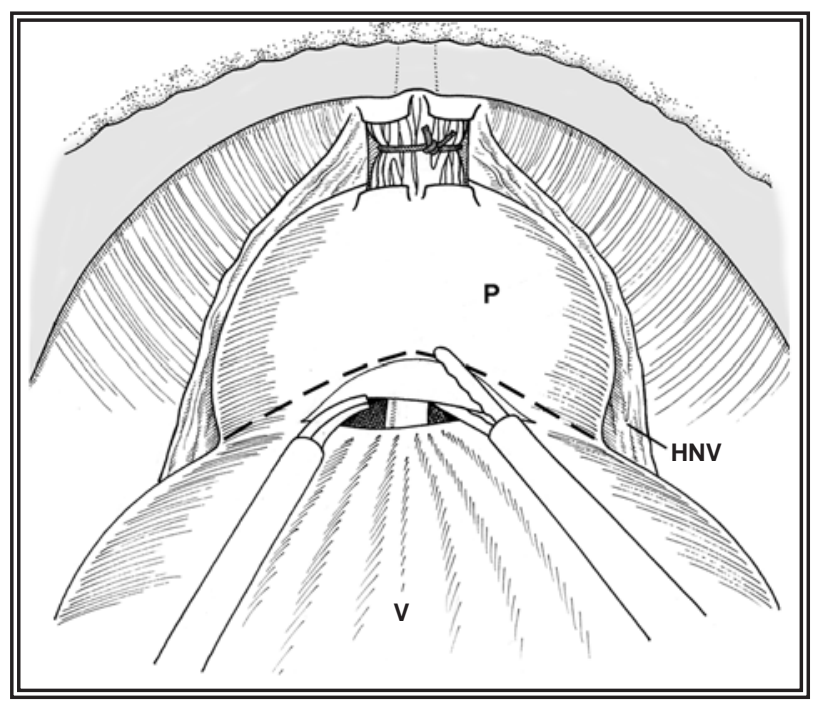

FIGURA 7. Disección anterior del cuello vesical. El cuello vesical se une a la próstata en una forma triangular. La disección se inicia en la posición de las 12 en la punta de este triángulo. La vista del catéter uretral es una referencia básica.

$P=$ próstata, $\mathrm{V}=$ vejiga $; \mathrm{HNV}=$ haces neurovasculares . 
Disección de las vesículas seminales e incisión de la fascia de Denonvillier

Después de la disección completa del cuello de la vejiga, el ayudante eleva anteriormente la próstata. Después de la disección de los deferentes (Fig. 8), se identifican fácilmente las vesículas seminales y se disecan completamente. La disección se realiza exclusivamente con las tijeras de ultrasonidos Ultracision o Sonosurge para evitar las lesiones a los haces neurovasculares que se encuentran próximas a las puntas de las vesículas seminales. Después de la disección de las vesículas seminales, el ayudante sujeta la ampolla derecha y la vesícula seminal derecha, el cirujano la ampolla izquierda y la vesícula seminal izquierda en una dirección craneolateral. Con esta maniobra, se desarrolla una "ventana" que alcanza desde la cara dorsal de la próstata a los pedículos prostáticos. Luego se corta la capa posterior de la fascia de Denonvillier y se visualiza el tejido graso prerrectal (Fig. 9). Se continúa la disección de forma roma y cortante lo más lejos posible hacia el vértice de la próstata, estrictamente en la línea media, para evitar las lesiones a los haces neurovasculares.

\section{Conservación de los haces neurovasculares}

La movilización de los haces neurovasculares comienza con la incisión de la fascia delgada que se encuentra por encima de la cara anterior-lateral de la próstata (fascia periprostática) durante la disección dorsal del cuello vesical. Esta inci-

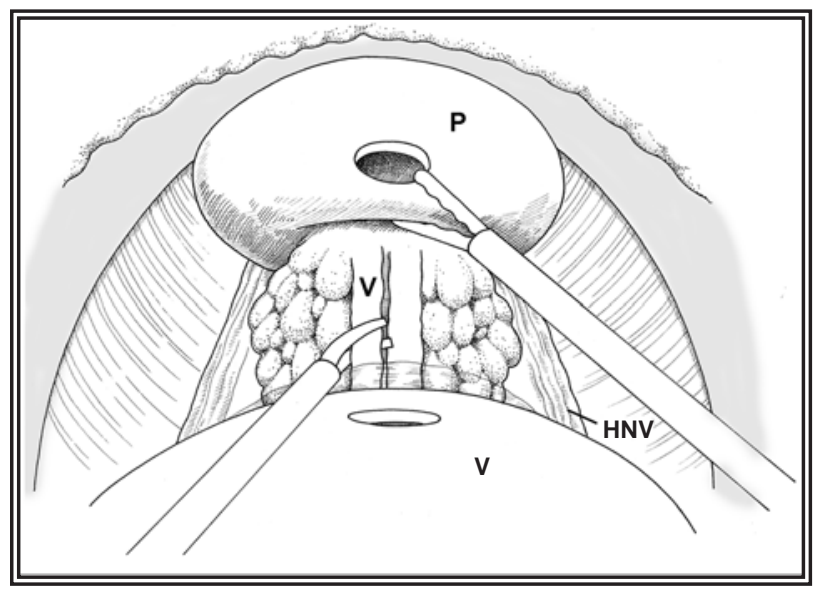

FIGURA 8 Corte de la porción ampular izquierda de los conductos deferentes. El ayudante eleva la próstata anteriormente en la dirección de la sinfisis.

$P=$ próstata, $\mathrm{V}=$ vejiga $; \mathrm{HNV}=$ haces neurovasculares,

$\mathrm{C}=$ ampolla del conducto deferente.

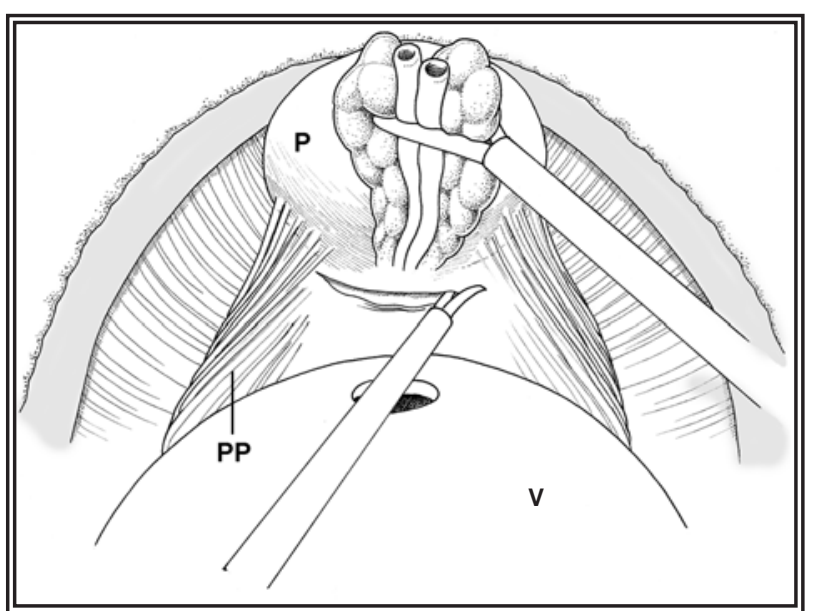

FIGURA 9. Incisión de la fascia de Denonvillier posterior. $P=$ próstata, $P P=$ pedículo prostático, $\mathrm{V}=$ vejiga .

sión se lleva hacia delante en dirección caudal. En muchos casos, es posible una disección roma de esta fascia delgada.

El proceso de conservación de los haces neurovasculares se continúa después de la disección completa de las vesículas seminales. Durante la preparación de los haces neurovasculares izquierdos, el ayudante retrae la próstata movilizada parcialmente hacia el lado derecho y viceversa. Los haces neurovasculares se fijan a la superficie dorsolateral de la próstata. Usando los dispositivos Ultracision o Sonosurge, los haces neurovasculares se separan completamente de la próstata durante la disección del pedículo prostático. El dispositivo se usa fundamentalmente como herramienta de disección y sólo cuando es absolutamente necesario se activa la energía térmica. Como alternativa, pueden ponerse grapas en vasos pequeños y dividirse entre endo-clips. Durante la separación del haz, los pedículos prostáticos se dividen meticulosamente con ayuda de los dispositivos Ultracision o Sonosurge. La disección comienza con el corte de los pedículos prostáticos craneales y se continúa caudalmente (Fig. 10). La conservación de los haces neurovasculares cerca del vértice de la próstata se realiza anterolateralmente. $\mathrm{El}$ ayudante tira de la próstata al lado derecho para la preparación del haz neurovascular izquierdo y viceversa.

Disección del plexo de la vena dorsal y disección apical

Se corta la uretra en el vértice como se describió previamente (Fig. 11) ${ }^{9-11}$. Debe evitarse estricta- 


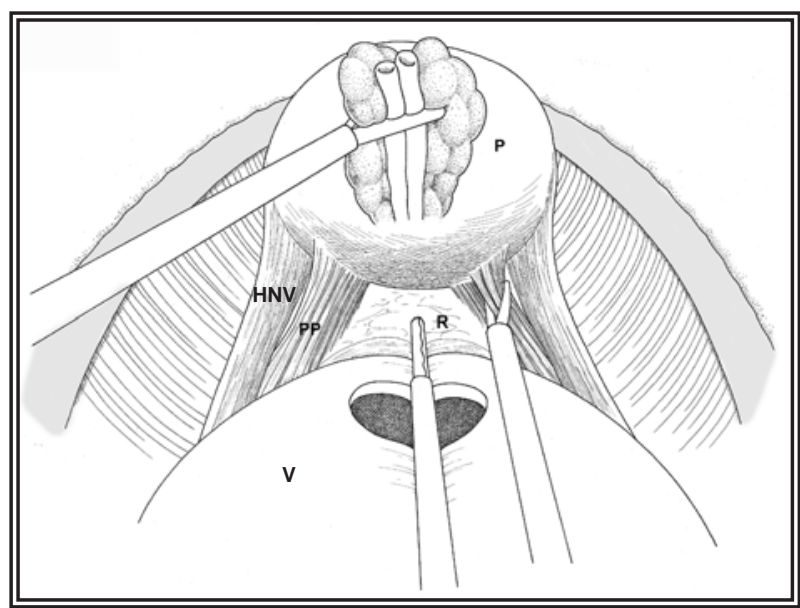

FIGURA 10. Disección de los pedículos prostáticos izquierdos con conservación de los haces neurovasculares. Durante la preparación del lado derecho, el cirujano retrae la próstata a la izquierda.

$P=$ próstata, $P P=$ pedículos prostáticos, $\mathrm{V}=$ vejiga; $\mathrm{HNV}=$ haces neurovasculares, $R=$ recto (tejido graso prerrectal).

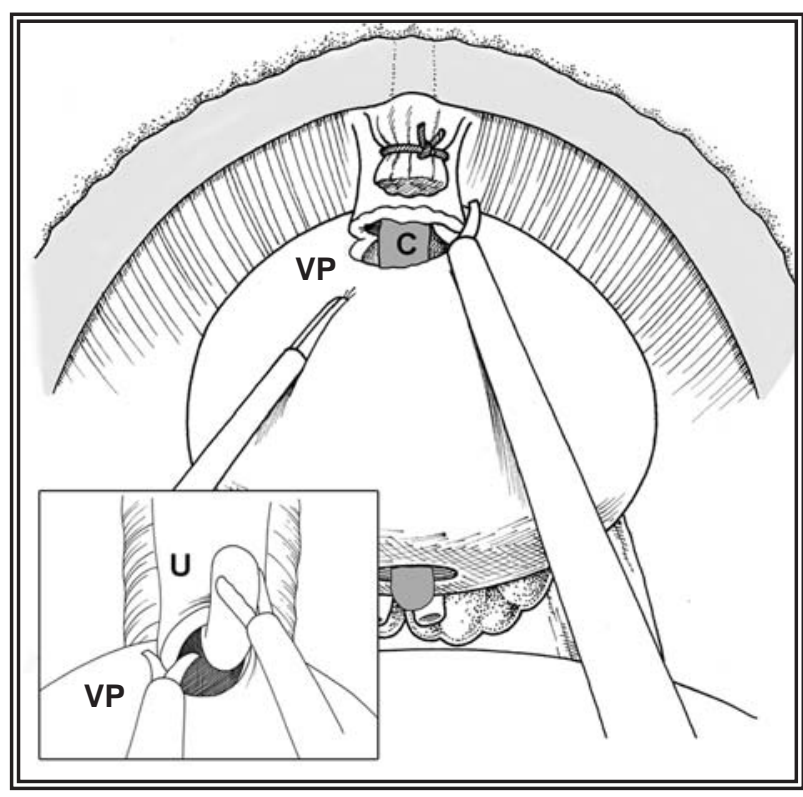

FIGURA 11. Disección apical: Corte del plexo de Santorini, la uretra ventral y luego la dorsal.

$\mathrm{VP}=$ vértice prostático, $\mathrm{C}=$ catéter, $\mathrm{U}=$ uretra .

mente la coagulación monopolar del muñón uretral para impedir el daño al esfinter externo estriado y a los haces nerviosos divergentes. En el caso de hemorragia menor en esta área, la presión de $\mathrm{CO}_{2}$ puede aumentarse temporalmente a 16-18 mmHg o se usa cuidadosamente coagulación bipolar.

Cuando la próstata se libera y diseca completamente de sus estructuras circundantes, se co- loca en una bolsa de recogida laparoscópica. La bolsa que contiene la pieza prostática se extrae parcialmente a través del lugar del trócar y se pinza con una pinza Kocher. Entonces se reinserta el trócar paralelo a la bolsa, que se encuentra en la fosa ilíaca izquierda (Fig.12).

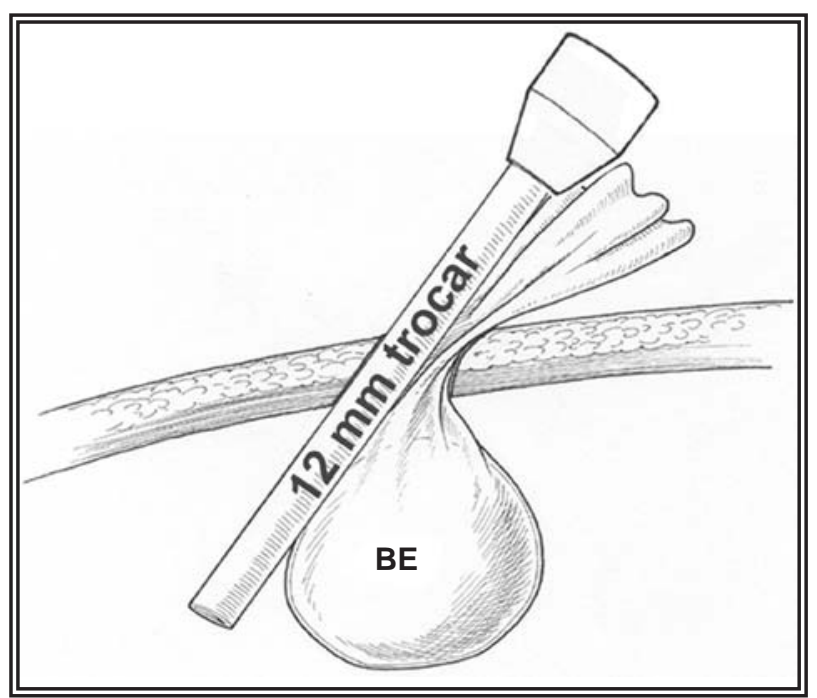

FIGURA 12. La bolsa de recogida endoscópica, que ha atrapado la próstata en la fosa ilíaca izquierda, protruye a través del trócar paralela al trócar de $12 \mathrm{~mm}$ y así permite la realización más fácil de la anastomosis. $B E=$ bolsa endoscópica.

Anastomosis uretrovesical (suturas interrumpidas)

La anastomosis se realiza con 9 suturas de Vicryl 2-0 interrumpidas con una aguja UR-6. Todos los puntos se realizan "de fuera a dentro" en el cuello de la vejiga y de "dentro a fuera" en la uretra. De esta manera, las suturas se atan siempre extraluminalmente (Fig. 13).

El primer punto comienza en la posición de las 8 (de revés) seguido por puntos en las posiciones 7,6 y 5 (hacia adelante) en el cuello vesical, de revés en la uretra. Después de cada punto uretral, tiene que retirarse hacia atrás el catéter para descartar la fijación por la sutura anastomótica. El punto de las 4 se realiza entonces hacia delante (cuello vesical) - hacia delante (uretra). Una vez terminada la circunferencia posterior, se coloca el catéter en la vejiga y se termina la anastomosis anterolateral y ventralmente. En el lado izquierdo, los puntos se realizan de revés-revés y en el lado derecho, hacia delante-delante.

Si durante la disección del cuello vesical no es factible una técnica de conservación del mismo, 


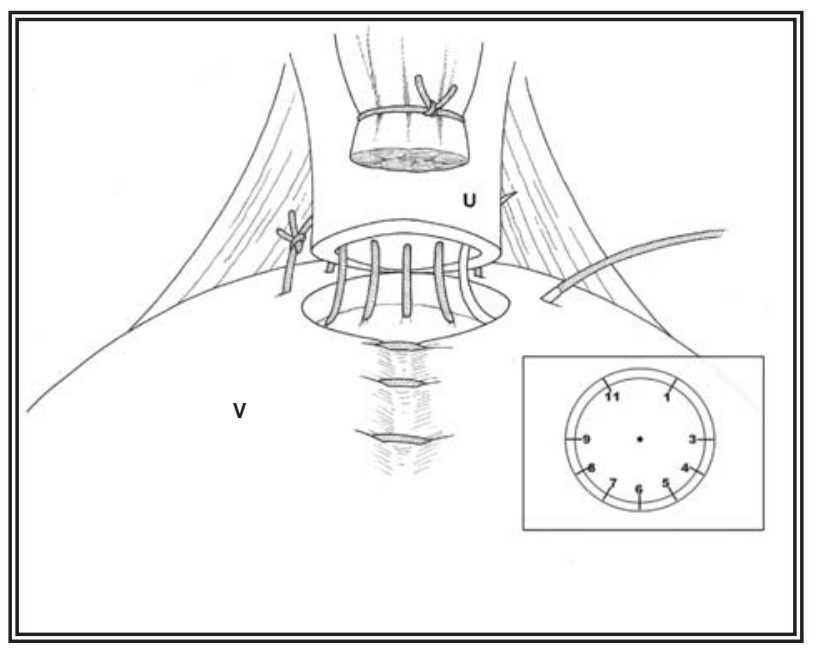

FIGURA 13. Anastomosis uretrovesical (suturas interrumpidas, Vicryl 2-0, aguja UR-6): Los puntos se realizan siempre "de fuera a dentro" en el cuello de la vejiga y "de dentro a fuera" en la uretra y siempre se atan extraluminalmente. Se ve la posición exacta de todos los puntos en el lado derecho de la figura. Observe que el cuello de la vejiga se ha reconstruido con 3 suturas interrumpidas en la posición de las 12 antes de la finalización de la anastomosis.

$\mathrm{V}=$ vejiga, $\mathrm{U}=$ uretra .

se hace una reconstrucción del cuello vesical en la posición de las 12, antes de colocarse los dos puntos finales en las posiciones $11 \mathrm{y} 1 \mathrm{de}$ la anastomosis (Fig. 13).

Finalmente, se comprueba la estanqueidad de la anastomosis llenando la vejiga con $200 \mathrm{ml} \mathrm{de}$ agua estéril. Al final del procedimiento, se coloca un catéter de drenaje Robinson $20 \mathrm{~F}$ en el espacio retropúbico. Finalmente, se extrae la bolsa laparoscópica con la pieza a través del trócar de $12 \mathrm{~mm}$ terminando así la cirugía. El drenaje se retira 24-48 horas después del procedimiento. A los cinco días de la intervención, se realiza una cistografía y si no hay fuga anastomótica, se retira el catéter uretral.

\section{MODIFICACIÓN CON CONSERVACIÓN DEL LIGAMENTO PUBOPROSTÁTICO}

Intentando mejorar los resultados de continencia precoz, desarrollamos la siguiente modificación técnica manteniendo intactos los ligamentos puboprostáticos. Inicialmente, se realiza una pequeña incisión de la fascia endopélvica para facilitar la ligadura del plexo de Santorini. No se incide toda la fascia endopélvica como se realiza en las técnicas de PRETEcn descritas previamente.
Luego se identifican los ligamentos puboprostáticos y se limpian de cualquier tejido graso suprayacente. Se usa luego un Vicryl 0 sobre una aguja CT-1 (ligeramente doblado hacia arriba para facilitar la maniobrabilidad) y se hace pasar por debajo de los ligamentos y por encima del plexo de Santorini de derecha a izquierda. Se guía la aguja de izquierda a derecha en el plano por debajo del complejo venoso dorsal y por encima de la pared uretral anterior justo caudal a los ligamentos puboprostáticos, teniendo cuidado de no tocar la uretra. Esta maniobra permite la ligadura del plexo sin afectación de los ligamentos puboprostáticos (Fig. 14). Comenzando desde el plano medial de los ligamentos, se realiza una incisión aguda bilateral caudalmente hacia el cuello de la vejiga y luego se desarrolla un plano entre la próstata y su delgada fascia suprayacente. El objetivo principal es desarrollar el plano correcto y finalmente, liberar la próstata de su "envoltura" dejando intacta la fascia periprostática, los ligamentos puboprostáticos y los haces nerviosos como una estructura continua (Fig. 15). La disección y la incisión de la próstata así como la anastomosis vesicouretral deben realizarse con mucho cuidado evitando atrapar los haces (Fig. 16) y/o los ligamentos, poniendo así en peligro el resultado final.

\section{REPARACIÓN SIMULTÁNEA DE HERNIAS}

En aproximadamente el 10\% de los pacientes operados con PRETE, hay necesidad de una reparación simultánea de una hernia inguinal unilateral

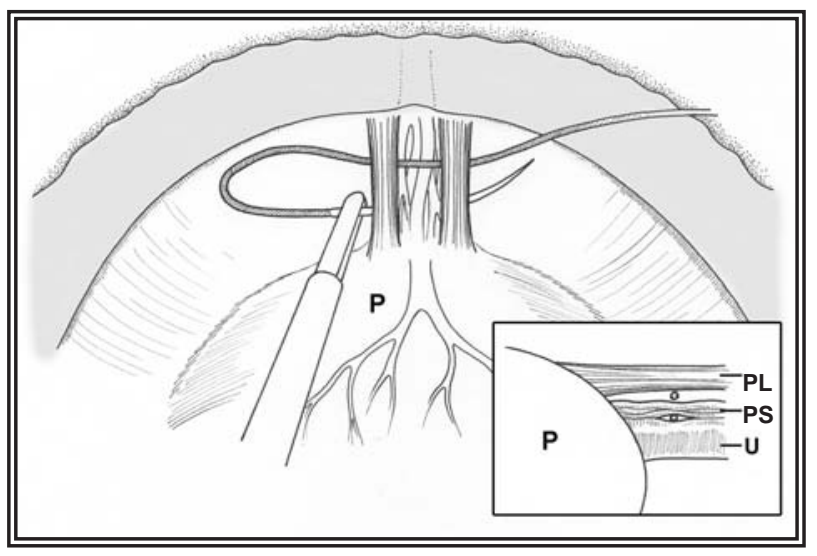

FIGURA 14. Maniobra técnica que permite la ligadura del plexo sin afectación de los ligamentos puboprostáticos. $P=$ próstata, $L P=$ ligamentos puboprostáticos, $P S=$ plexo de Santorini, $U$ = uretra . 


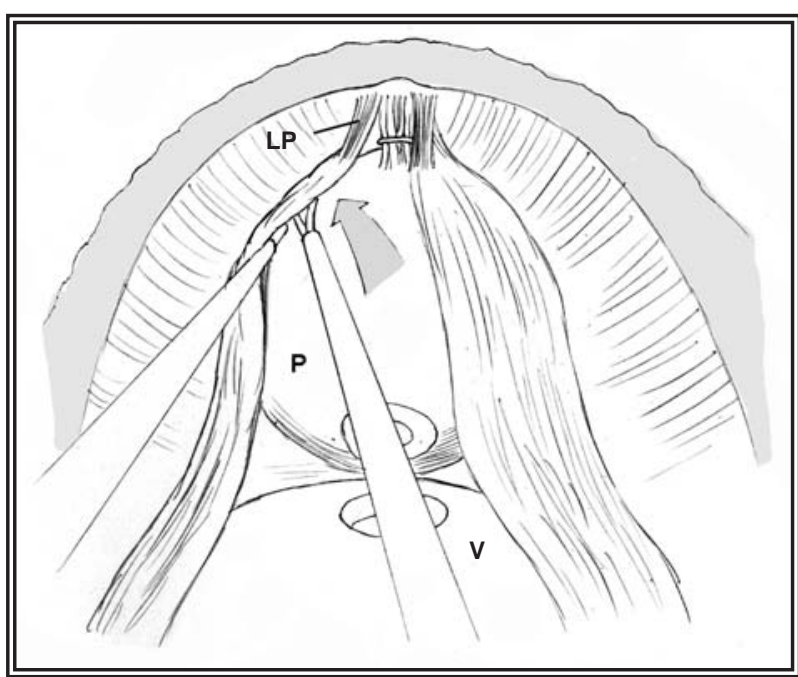

FIGURA 15. Disección roma y cortante anterolateral de la próstata desde la fascia periprostática, los ligamentos puboprostáticos y los haces nerviosos.

$V=$ vejiga, $P$ = próstata, $L P=$ ligamentos puboprostáticos.

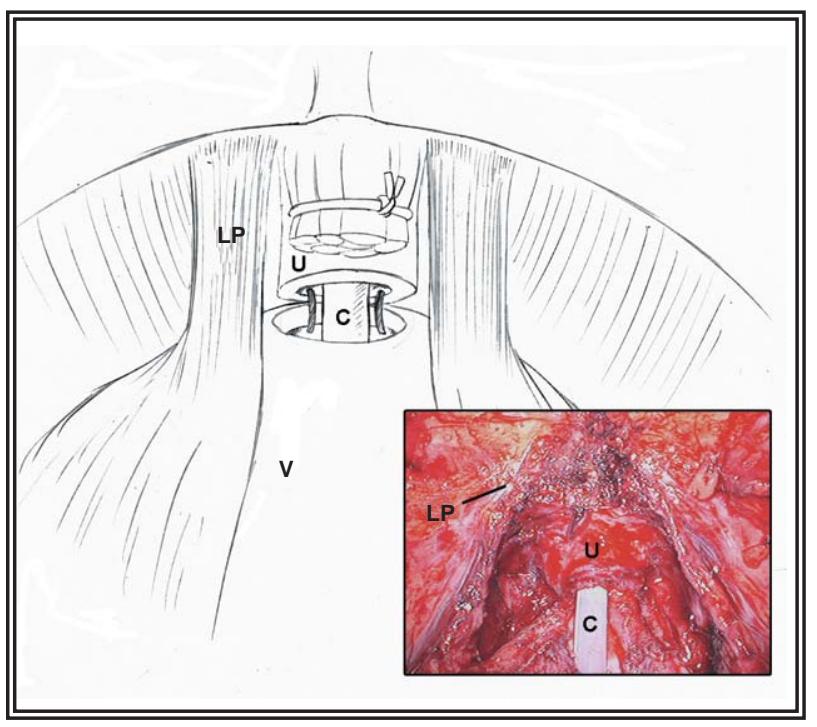

FIGURA 16. Se ha concluido la parte dorsal de la anastomosis. La anastomosis vesicouretral debe realizarse con mucho cuidado evitando atrapar a los haces y/o los ligamentos. Vista endoscópica con identificación clara de los ligamentos puboprostáticos, la uretra, el catéter y la anastomosis dorsal.

$V=$ vejiga,$C=$ catéter, $L P=$ ligamentos puboprostáticos, $U=$ uretra .

o bilateral. Hay diferentes técnicas para colocar la malla en el espacio preperitoneal para cubrir todos los posibles defectos de hernia. Nosotros preferimos una técnica totalmente extraperitoneal, que usa el principio de reparación de hernia sin tensión, tapando todo el orificio miopectíneo con una larga pieza de malla. La malla es de 10 x $15 \mathrm{~cm}$; se corta en la parte media y se hace un pequeño agujero en la malla para proporcionar espacio suficiente para el cordón espermático. La división dentro de la malla se cubre con un colgajo $(6$ x 5 $\mathrm{cm})$. Los puntos esenciales de esta técnica son elevar el cordón espermático al lado del defecto de la hernia inguinal y colocar la malla de polipropileno preparada alrededor del cordón espermático, para cubrir los orificios herniarios y todo el espacio desde la sínfisis del pubis en la línea media a la pared abdominal lateralmente (Fig. 17). No se necesitan grapas ni suturas para fijar la malla; la malla se ancla y se fija a la pared abdominal mediante la presión intrabdominal exclusivamente $^{12}$. El cierre del defecto peritoneal (si lo hay) después de colocar la malla es esencial para evitar la exposición de la malla a los órganos intrabdominales. Debe evitarse el contacto directo de la malla con el intestino.

\section{NUESTROS RESULTADOS}

Entre diciembre de 2001 y agosto de 2005, hemos realizado aproximadamente 900 PRETE

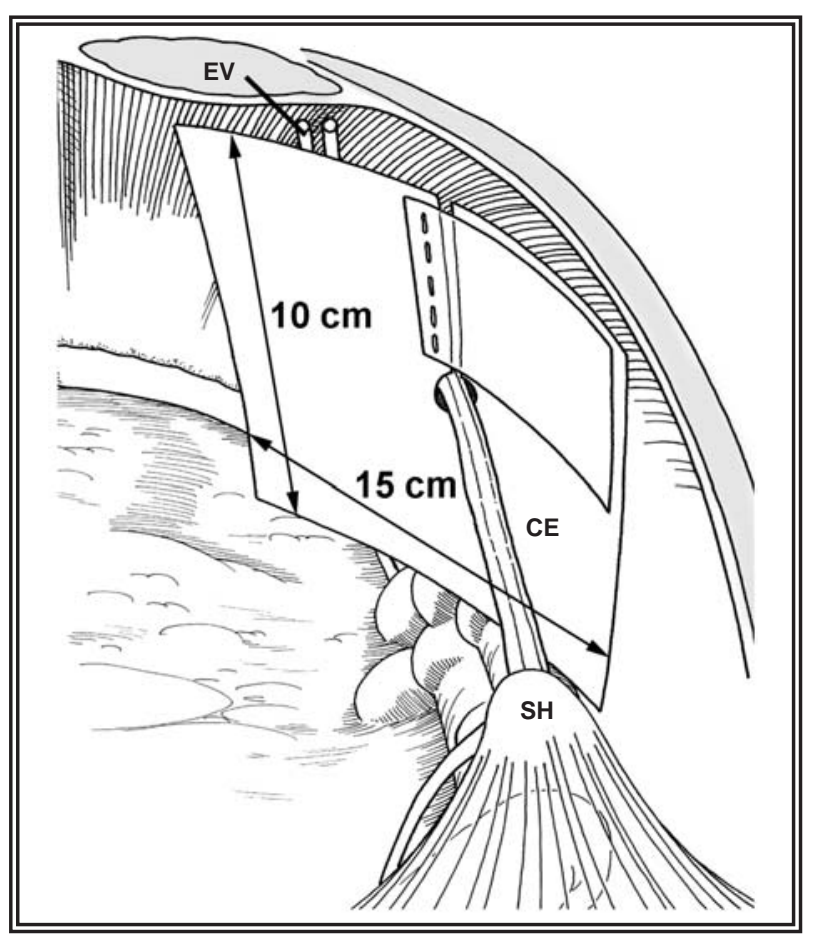

FIGURA 17. En el caso de la hernia inguinal simultánea, se coloca una malla de polipropileno de $10 \times 15 \mathrm{~cm}$ en el espacio preperitoneal que cubre los orificios herniarios tanto directo como indirecto al final de la prostatectomía. $\mathrm{VE}$ = vasos epigástricos, $\mathrm{CE}=$ cordón espermático; SH = saco herniario. 
consecutivas. Los resultados de nuestros 700 casos iniciales se han comunicado recientemen$\mathrm{te}^{2}$. En pocas palabras, un total de 700 pacientes consecutivos se sometieron a PRETE. La media de edad era de 63,4 (42-77) años. El nivel medio preoperatorio de PSA era de 10,7 (1,4-82 ng/ml) ng/ml. Un total de 206 pacientes $(29,4 \%)$ tenían antecedentes de cirugía abdominal baja o pélvica previas, incluidas hernioplastia inguinal con colocación de malla en 11 pacientes. Cuarenta y tres pacientes $(6,1 \%)$ tenían antecedentes de intervención prostática (RTUP, HIFU, prostatectomía de tipo Millin, radioterapia).

$\mathrm{El}$ promedio de tiempo quirúrgico incluyendo a todos los pacientes sometidos a disección de ganglios linfáticos pélvicos y/o herniorrafia extraperitoneal con colocación de malla fue de 151 min (50-320 min). El volumen prostático medio era de 51,1 g (rango, 12-100 g). No fue necesaria ninguna conversión a cirugia abierta. La media de pérdida de sangre fue de $220 \mathrm{ml}$ (rango, de 50$1.000 \mathrm{ml}$ ). Un total de seis pacientes precisaron transfusiones sanguíneas (tasa de transfusión del 0,9\%). La media global de tiempo de cateterización postoperatorio de la vejiga fue de 6,2 días.

Se definió la continencia plena como la ausencia de necesidad de pañales. Se clasificó la fuga de orina ocasional (1 a 2 pañales al día) como incontinencia de estrés mínima durante el día con actividad normal, incluida actividad deportiva moderada como el caminar. Después de un seguimiento posoperatorio de seis meses, el 83,8\% $(n=419)$ de 500 pacientes eran completamente continentes, el 10,4\% ( $n=52)$ tenían una incontinencia de estrés mínima y el 5,8\% $(n=29)$ precisaban más de 2 pañales al día. Estos datos se comparan igualmente con los resultados publicados previamente para la PRL. Guilloneau et al. y Türk et al. comunicaron tasas de continencia completa después de seis meses del $76 \%$ y el $86 \%$, respectivamente $^{13,14}$. Después de 12 meses, el 92\%, el 6\% y el $2 \%$ de nuestros pacientes eran completamente continentes, necesitaban 1-2 pañales al día o 2 o más pañales al día, respectivamente.

Se realizó PRETE con conservación de nervios (PRETEcn) en 185/700 (26,4\%) pacientes potentes preoperatoriamente ya fuese unilateralmente ( $\mathrm{n}=114$ ) o bilateralmente $(\mathrm{n}=71)$. Se dispuso de datos de seguimiento a los seis meses para 66 pacientes con PRETEcn unilateral y 34 pacientes con PRETEcn bilateral. Se comunicaron erecciones suficientes para una relación sexual en 8/66 $(12,1 \%)$ y $16 / 34(47,1 \%)$ de los pacientes, respectivamente. Aunque el seguimiento es demasiado corto para extraer conclusiones definitivas, es obvio que un planteamiento conservador en PRETE es factible y reproducible. Las tasas de potencia después de la prostatectomía radical retropúbica abierta conservadora de nervios unilateral y bilateral varían considerablemente entre los diferentes centros y alcanzan hasta el $86 \%$ a los 12 meses (pacientes más jóvenes, conservación de nervios bilaterales, uso de inhibidores de la PDE5 incluido) ${ }^{15-17}$. En general, las tasas de potencia a corto plazo deben discutirse con cuidado, porque la vuelta de la función sexual después de prostatectomía radical conservadora de nervios unilateral y bilateral puede tardar hasta 48 meses $^{18}$.

Los estadios patológicos postoperatorios fueron pT2 en 388 (55,4\%), pT3 en 308 (44\%) y pT4 en $4(0,6 \%)$ pacientes. Se encontraron márgenes quirúrgicos positivos en el 10,8\% de los pacientes con un tumor pT2 y en el $31,2 \%$ de los pacientes con un tumor pT3. De los 266 pacientes, sometidos a disección de los ganglios linfáticos pélvicos, el 5,3\% tenían metástasis en los ganglios linfáticos pélvicos. Como comunicamos los resultados de seguimiento a corto plazo, no podemos comentar el resultado oncológico a largo plazo. Sin embargo, los resultados en cuanto a control del cáncer parecen equivalentes a los de la cirugía abierta. Wieder y Soloway obtuvieron una tasa global de márgenes quirúrgicos positivos después de una prostatectomía radical abierta de un $28 \%{ }^{19}$. Los resultados para la PRL van entre un $6,9 \%$ para los tumores pT2a y un $34 \%$ para los tumores pT3b en una evaluación oncológica de 1.000 casos en el Instituto Montsouris ${ }^{5}$.

Entre las complicaciones intraoperatorias hubo lesiones rectales $(0,6 \%)$, reparadas laparoscópicamente con una sutura de 2 capas y lesión de los vasos epigástricos producida por colocación del trócar $(1,1 \%)$ tratadas con coagulación, colocación de grapas o suturas. Observamos complicaciones posoperatorias que precisaron reintervención en el 2\% (14 pacientes) de los casos, incluidos linfoceles sangrantes y/o sinto- 
máticos. Debido a la incidencia posoperatoria de linfoceles, hemos modificado nuestra estrategia quirúrgica desde la coagulación bipolar a la colocación de grapas en los vasos linfáticos.

\section{DISCUSIóN}

La PRL y la PRETE, aunque se consideran entre los procedimientos laparoscópicos técnicamente más exigentes en la urología, se han establecido como procedimientos estándar realizados habitualmente como tratamiento de primera línea para el cáncer de próstata localizado en centros especializados. Entre las principales ventajas asociadas a la laparoscopia mínimamente invasiva están, por ejemplo, menores tasas de sangrado intraoperatorio, menos dolor postoperatorio, un período más corto de cateterización uretral y una estancia hospitalaria más corta. El abordaje transperitoneal de un órgano extraperitoneal puede producir complicaciones intraperitoneales como lesiones intestinales, peritonitis, íleo posoperatorio, adherencias peritoneales, sangrado intraperitoneal o fuga intraperitoneal de orina ${ }^{1-6}$ por tanto, defendemos el uso de la vía extraperitoneal para la realización del procedimiento laparoscópico.

Desde que comunicamos nuestra experiencia inicial con 70 PETRE, hemos mejorado de forma continua y hemos estandarizado nuestra técnica. Hoy, nuestra experiencia se basa en una gran serie de 700 pacientes, que se sometieron a prostatectomía radical mínimamente invasiva usando este abordaje retropúbico completamente extraperitoneal. No hay criterios específicos de selección de pacientes, ni contraindicaciones específicas. Los procedimientos quirúrgicos abdominales previos no afectan a los tiempos quirúrgicos globales ni a las tasas de complicaciones ${ }^{22}$.

En conclusión, los resultados de esta gran serie de 700 pacientes son prometedores. En nuestro estudio basado en la ejecución por parte de cinco cirujanos diferentes (incluidos 2 residentes sin experiencia previa en prostatectomía abierta) usando el mismo abordaje, la tasa de transfusiones fue del $0,9 \%(n=6)$. No fue necesaria ninguna conversión a cirugía abierta y el tiempo quirúrgico medio fue de 151 minutos y por tanto, sustancialmente más corto que las series comparables de PRL (203 y 218 min) ${ }^{20,21}$. La morbilidad peroperatoria es muy baja y los resultados funcionales a corto plazo y el control del cáncer son favorables. Nuestros resultados demuestran que la PRETE/PRETEcn puede realizarse con igual eficacia y los resultados preliminares son comparables a los de la prostatectomía radical abierta estándar así como la PRL transperitoneal mientras que se evitan completamente las complicaciones intraperitoneales.

Agradecimientos: Los autores agradecen la ayuda del Sr. Jens Mondry (Director de Moonsoft, Alemania) para elaborar las Figuras 1-4 y el Sr. Gottfried Müller para elaborar las Figuras 5-17.

\section{REFERENCIAS}

c1. Rassweiler J, Schulze M, Teber D, Seemann O, Frede T. Laparoscopic radical prostatectomy: functional and oncological outcomes. Curr Opin Urol. 2004;14(2):75-82.

c2. Stolzenburg JU, Rabenalt R, Do M, Ho K, Dorschner W, Waldkirch E, et al. Endoscopic Extraperitoneal Radical Prostatectomy (EERPE) - oncological and functional results after 700 procedures. J Urol. 2005;174(4Pt1): 1271-1275.

c3. Anastasiadis AG, Salomon L, Katz R, Hoznek A, Chopin D, Abbou C. Radical retropubic versus laparoscopic prostatectomy: a prospective comparison of functional outcome. Urology. 62(2):292-297.

c4. Brown JA, Garlitz C, Gomella LG, Hubosky SG, Diamond SM, McGinnis D. Pathologic comparison of laparoscopic versus open radical retropubic prostatectomy specimens. Urology. 2003;62(3):481-486.

c5. Guillonneau B, el-Fettouh H, Baumert H, Cathelinea X, Doublet JD, Fromont G, et al. Laparoscopic radical prostatectomy: oncological evaluation after 1,000 cases at Montsouris Institute. J Urol. 2003;169(4):1261-1266.

c6. Steinberg AP, Gill IS. Laparoscopic prostatectomy: a promising option in the treatment of prostate cancer. Cleve Clin J Med. 2004;71(2):78.

c7. Raboy A, Ferzli G, Albert P. Initial experience with extraperitoneal endoscopic radical retropubic prostatectomy. Urology. 1997;50(6):849-853.

c8. Bollens R, Vanden Bossche M, Roumeguere T, Damoun A, Ekane S, Hoffmann P, et al. Extraperitoneal laparoscopic radical prostatectomy. Results after 50 cases. Eur Urol. 2001;40(1):65-69.

19. Stolzenburg JU, Do M, Pfeiffer H, König F, Aedtner B, Dorschner W. The endoscopic extraperitoneal radical prostatectomy (EERPE): technique and initial experience. World J Urol. 2002;20(1):48-55.

10. Stolzenburg JU, Do M, Rabenalt R, Pfeiffer H, Horn L, Truss MC, et al. Endoscopic Extraperitoneal Radical Prostatectomy (EERPE)-initial experience after 70 procedures. J Urol. 2003;169(6):2066-2071.

11. Stolzenburg JU, Truss MC, Do M, Rabenalt R, Pfeiffer H, Dunzinger $\mathrm{M}$, et al. Evolution of endoscopic extraperitoneal radical prostatectomy (EERPE)-technical improvements and development of a nerve-sparing, potency-preserving approach. World J Urol. 2003;21(3):147-152.

12. Stolzenburg JU, Rabenalt R, Dietel A, Do M, Pfeiffer H, Schwalbe $\mathrm{S}$, et al. Hernia repair during endoscopic (laparoscopic) radical prostatectomy. J Laparoendosc Adv Surg Tech A. 2003;13(1):27-31. 
13. Guillonneau B, Rozet F, Barret E, Cathelineau X, Vallancien G. Laparoscopic radical prostatectomy: assessment after 240 procedures. Urol Clin North Am. 2001; 28(1):189-202.

14. Türk I, Deger IS, Winkelmann B, Roigas J, Schonberger B, Loening SA. Laparoscopic radical prostatectomy. Experience with 145 interventions. Urologe A. 2001 May; 40(3): 199-206.

15. Noldus J, Michl U, Graefen M, et al. Patient-reported sexual function after nerve-sparing retropubic radical prostatectomy. Eur Urol. 2002;42(2): 118-124.

16. Walsh PC. Radical prostatectomy for localized prostate cancer provides durable cancer control with excellent quality of life: a structurated debate. J Urol. 2000;163(6): 1802-1807.

17. Kundu SD, Roehl KA, Eggener SE, Antenor JAV, Han M, Catalona WJ. Potency, continence and complications in 3477 consecutive radical retropubic prostatectomies. J Urol. 2004;172(6 Pt 1):2227-2231.

18. Rabbani F, Patel MI, Scardino PT. Time course of recovery of potency after bilateral nerve sparing radical prostatectomy. J Urol. 171(4, suppl.), A1178, 2004.
19. Wieder JA, Soloway MS. Incidence, etiology, location, prevention and treatment of positive surgical margins after radical prostatectomy for prostate cancer. J Urol. 1998; 160(2):299-315.

20. Rassweiler J, Seemann O, Schulze M, Teber D, Hatzinger M, Frede T. Laparoscopic versus open radical prostatectomy: a comparative study at a single institution. J Urol. 2003;169(5): 1689-1693.

21. Guillonneau B, Rozet F, Cathelineau X, Lay F, Barret E, Doublet JD, et al. Perioperative complications of laparoscopic radical prostatectomy: the Montsouris 3-year experience. J Urol. 2002 ; 167(1):51-56.

22. Stolzenburg JU, Ho KM, Do M, Rabenalt R, Dorschner W, Truss MC. Impact of previous surgery on endoscopic extraperitoneal radical prostatectomy. Urology. 2005;65(2):325-231.

Dr. Jens-Uwe Stolzenburg MD PhD

University of Leipzig, Department of Urology, Liebigstraße 20, 04103 Leipzig, Alemania E-mail: stolj@medizin.uni-leipzig.de

(Trabajo recibido el 16 de septiembre 2005) 